\title{
Rayleigh-Taylor instability of viscous liquid films under a temperature-controlled inclined substrate
}

\author{
Youchuang Chao $\odot,{ }^{1, *}$ Lailai Zhu $\odot,{ }^{2, \dagger}$ and Hao Yuan ${ }^{3}$ \\ ${ }^{1}$ Max Planck Institute for Dynamics and Self-Organization, 37077 Göttingen, Germany \\ ${ }^{2}$ Department of Mechanical Engineering, National University of Singapore, 117575, Singapore \\ ${ }^{3}$ School of Life Science and Engineering, Southwest Jiaotong University, 610031 Chengdu, China
}

(Received 15 January 2021; accepted 26 May 2021; published 14 June 2021)

\begin{abstract}
We study the Rayleigh-Taylor instability of gravity-driven viscous liquid films flowing under a uniformly heated or cooled inclined substrate. The long-wave assumption is adopted to derive the evolution equation of the film, which is characterized by five dimensionless parameters including Marangoni number Ma, Biot number Bi, Reynolds number Re, Weber number We, and the inclination angle $\alpha$ of the substrate. Based on the long-wave equation, we systematically examine the temporal and spatiotemporal stability of the system. Temporal stability analysis shows that the thermocapillary stress reinforces the Rayleigh-Taylor instability of a heated film but counteracts the instability of a cooled film, as verified by the numerical solutions of linearized Navier-Stokes equation. In particular, this instability can be completely inhibited if a composite Marangoni number $\mathrm{Ma}^{*}$ is below a critical value $\mathrm{Ma}_{1}^{*}$. We further perform a spatiotemporal stability analysis to delineate the absolute and convective nature of the temporally unstable system. We find that the thermocapillary stress in the heated film enhances the absolute instability and suppresses the convective instability. The trend reverses for a cooled film that is featured by suppressed absolute instability and enhanced convective instability. More importantly, the transition between the absolute and convective instability can be characterized by another critical value, $\mathrm{Ma}_{2}^{*}$, beyond which the flow stability will be triggered from the convectively into the absolutely unstable. The predictions from linear stability analysis are confirmed by numerical solutions of the full long-wave evolution equation.
\end{abstract}

DOI: 10.1103/PhysRevFluids.6.064001

\section{INTRODUCTION}

Rayleigh-Taylor (RT) instability occurs when a fluid rests above a lighter one in a gravitational field or in a system which is accelerating in the direction from the lighter to the denser fluid [1-3]. As a fundamental interfacial phenomenon, the RT instability is ubiquitous in our everyday life, such as the dripping of droplets from condensed vapor under bathroom ceilings, as well as in nature, such as the formation of mushroom clouds from volcanic eruptions and fingerlike patterns in granular flows [4]. In certain engineering applications, however, this instability is undesired and detrimental. For instance, the RT instability emerging in coating processes may result in the irregular coating of paints

\footnotetext{
*ychao@connect.hku.hk

†lailai_zhu@nus.edu.sg
}

Published by the American Physical Society under the terms of the Creative Commons Attribution 4.0 International license. Further distribution of this work must maintain attribution to the author(s) and the published article's title, journal citation, and DOI. Open access publication funded by the Max Planck Society. 
and even lead to droplet detachments in the case of thick coatings [5]. Another example is related to the nuclear fusion reaction, where instability-induced droplets falling into the high-temperature plasma could quench the processes of fuel compression and confinement $[6,7]$. Therefore, controlling and suppressing RT instability is critically important in a variety of engineering applications. To achieve this goal, a growing number of approaches have been proposed, e.g., by utilizing an electric field [8-10], a high-frequency mechanical oscillation [11-14], a rotating magnetic field [15-17], and recently proposed geometric features such as substrate curvature $[18,19]$ and spatial confinement [20]. Apart from these strategies, exploiting the thermocapillary stress developed by heating or cooling the film represents another important and facile method to control this instability.

In a canonical scenario, a liquid film is coated beneath a planar substrate [21,22]. Without additional complexities, the instability of the film is determined by the competition between the destabilizing gravitational force that pulls the liquids down, and the stabilizing capillary force that flattens the liquid-gas interface [23]. The surface-tension gradient brought by temperature variations at the interface can induce an unbalanced tangential stress, known as thermocapillary or Marangoni stress, that is capable of modulating RT instability [24,25]. As such, suppression of this instability has been achieved by applying a temperature gradient perpendicular to a horizontal film. For instance, Deissler and Oron theoretically found that cooling the substrate could produce a steady, nonruptured suspending film, which was explained by minimizing an appropriate Lyapunov functional of the system [26]. Afterwards, Burgess et al. experimentally demonstrated that heating the liquid-gas interface could also prevent the liquid droplets dripping from the ceiling when the produced temperature gradient was above a critical value [27]. More recently, the experimental finding of Burgess et al. [27] was theoretically explained by Alexeev and Oron [28], where the time-dependent Navier-Stokes (NS) and long-wave evolution equations in both two-dimensional and three-dimensional situations were solved and analyzed. In the above-mentioned studies [26-28], the substrate was horizontally placed. However, how the thermocapillary stress controls or modifies the RT instability for the situation of an inclined substrate remains unclear.

Unlike a horizontal substrate, the fluid gravity in the inclined case has not only a component perpendicular to the surface but also a tangent component that is responsible for the spatiotemporal stability of the liquid film. Recent studies on the isothermal film have shown that when the inclination from the horizontal direction is increased beyond a critical angle, the flow will transit from absolute into convective instability. This transition is found to be a crucial factor to determine whether the droplet will detach from the substrate or not. For instance, Brun et al. [5] demonstrated that droplet dripping could be suppressed for a sufficiently inclined substrate; this phenomenon cannot be explained by traditional temporal linear stability analysis but can be rationalized as a transition from an absolute to a convective instability in the context of spatiotemporal analysis $[5,29]$. These studies highlight the necessity of considering absolute and convective instability when dealing with the RT instability of liquid films beneath an inclined substrate. In this paper we revisit the role of thermocapillary stress on the RT instability of nonisothermal films under a substrate with varying temperature but focus on the case of an inclined substrate (see Fig. 1), where the spatiotemporal stability is comprehensively investigated.

The rest of the paper is organized as follows. In Sec. II we present the physical model and derive the long-wave evolution equation for the thickness of the liquid film. In Sec. III we perform linear stability analysis, including both temporal and spatiotemporal analysis. Numerical solutions of the full evolution equation are presented in Sec. IV. In Sec. V, we give the conclusions and discussion of this work.

\section{PROBLEM FORMULATION}

\section{A. Governing equations}

We consider the dynamics of a Newtonian viscous liquid film with constant dynamic viscosity $\mu$ and density $\rho$ beneath an inclined substrate with a uniform and constant temperature $T_{\mathrm{s}}$. Here 


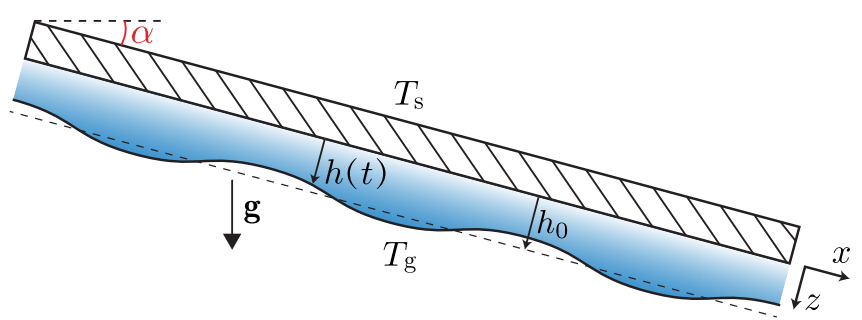

FIG. 1. A viscous liquid film of initial thickness $h_{0}$ under a temperature-controlled (uniformly heated or cooled) substrate that is tilted from the horizontal direction by an angle $\alpha$. The temperature maintained at the substrate and of the gas is denoted by $T_{\mathrm{s}}$ and $T_{\mathrm{g}}$, respectively.

$T_{\mathrm{s}}$ is generally not equal to the ambient gas temperature $T_{\mathrm{g}}$. The inclination angle of the substrate from the horizontal is defined as $\alpha$, where $\alpha=0$ corresponds to the horizontal case, as shown in Fig. 1. The flow is described in a two-dimensional Cartesian coordinate system $(x, z)$, where $x$ and $z$ coordinates are in the directions along and normal to the solid substrate, respectively. The ambient gas is passive and inviscid so that its motion is neglected. The instantaneous location of the film interface is denoted by $h(x, t)$, with an unperturbed thickness of $h_{0}$. The dynamics of the flow are governed by the incompressible NS and the energy equations,

$$
\begin{aligned}
u_{x}+w_{z} & =0, \\
u_{t}+u u_{x}+w u_{z} & =-\frac{1}{\rho} p_{x}+\frac{\mu}{\rho}\left(u_{x x}+u_{z z}\right)+g \sin \alpha, \\
w_{t}+u w_{x}+w w_{z} & =-\frac{1}{\rho} p_{z}+\frac{\mu}{\rho}\left(w_{x x}+w_{z z}\right)+g \cos \alpha, \\
T_{t}+u T_{x}+w T_{z} & =\kappa\left(T_{x x}+T_{z z}\right),
\end{aligned}
$$

where $u$ and $w$ represent the velocities in the $x$ and $z$ directions, respectively. Here, $p, T$, and $\mathbf{g}=$ $(g \sin \alpha, g \cos \alpha)$ indicate the pressure, temperature, and gravitational acceleration, respectively; $\kappa=$ $\lambda / \rho c_{p}$ is the thermal diffusivity of the liquid, with $\lambda$ and $c_{p}$ denoting the thermal conductivity and the specific heat capacity of the liquid, respectively. Without any further specification, all indices in the variables indicate the associated partial derivatives.

At the substrate surface $(z=0)$, a no-slip, no-penetration condition and a constant surface temperature are applied:

$$
u=0, \quad w=0, \quad T=T_{\mathrm{s}} .
$$

When the substrate is uniformly heated, $T_{\mathrm{s}}>T_{\mathrm{g}}$, and when the substrate is uniformly cooled, $T_{\mathrm{s}}<T_{\mathrm{g}}$.

The balances of normal stresses and of tangential shear stresses at the liquid-air interface $(z=h)$ yield, respectively,

$$
\begin{aligned}
-p+\frac{2 \mu}{1+h_{x}^{2}}\left[u_{x}\left(h_{x}^{2}-1\right)-h_{x}\left(u_{z}+w_{x}\right)\right] & =\frac{\sigma}{\left(1+h_{x}^{2}\right)^{3 / 2}} h_{x x}, \\
\frac{\mu}{\left(1+h_{x}^{2}\right)^{1 / 2}}\left[\left(1-h_{x}^{2}\right)\left(u_{z}+w_{x}\right)+2 h_{x}\left(w_{z}-u_{x}\right)\right] & =\frac{\partial \sigma}{\partial T}\left(T_{x}+h_{x} T_{z}\right),
\end{aligned}
$$

where $\sigma$ is the surface tension of the liquid-air interface. Normally, the tension $\sigma$ of most fluids is assumed to decrease linearly with the increase of temperature [30] as

$$
\sigma=\sigma_{0}-\sigma_{T}\left(T-T_{\mathrm{g}}\right)
$$


where $\sigma_{0}$ is the surface tension at the reference point $T_{\mathrm{g}}$, chosen as the temperature of the ambient gas far away from the film, and $\sigma_{T}=-(d \sigma / d T)>0$ is the surface-tension parameter.

At the liquid-air interface $(z=h)$, the heat transfer between the liquid and the ambient gas is given by the Newton's law of cooling [31],

$$
-\frac{\lambda}{\left(1+h_{x}^{2}\right)^{1 / 2}}\left(-h_{x} T_{x}+T_{z}\right)=h_{\mathrm{g}}\left(T-T_{\mathrm{g}}\right),
$$

where $h_{\mathrm{g}}$ is the rate of convective heat transfer from the liquid to the ambient gas. The system is closed by imposing the kinematic boundary condition at $z=h$, which reads

$$
h_{t}+u h_{x}-w=0 \text {. }
$$

\section{B. Nondimensionalization}

To nondimensionalize the above governing system, we apply the following set of scalings:

$$
\begin{aligned}
(u, w) & =U_{0}(U, W), \quad(x, z, h)=h_{0}(X, Z, H), \\
p & =\frac{\mu U_{0}}{h_{0}} P, \quad t=\frac{h_{0}}{U_{0}} \tau, \quad T-T_{\mathrm{g}}=\left(T_{\mathrm{s}}-T_{\mathrm{g}}\right) \theta,
\end{aligned}
$$

where $U_{0}=\rho g h_{0}^{2} \sin \alpha / \mu$ is the characteristic velocity scale, given by the balance between the $x$ component of gravity and the viscous force, and $T_{\mathrm{s}}-T_{\mathrm{g}}$ is the characteristic temperature scale, defined as the temperature difference between the substrate surface and the ambient gas.

After substituting the dimensionless variables in Eq. (7) into Eqs. (1)-(6), the nondimensionalized governing equations are obtained:

$$
\begin{aligned}
U_{X}+W_{Z} & =0, \\
\operatorname{Re}\left(U_{\tau}+U U_{X}+W U_{Z}\right) & =-P_{X}+U_{X X}+U_{Z Z}+1, \\
\operatorname{Re}\left(W_{\tau}+U W_{X}+W W_{Z}\right) & =-P_{Z}+W_{X X}+W_{Z Z}+\cot \alpha, \\
\operatorname{Pe}\left(\theta_{\tau}+U \theta_{X}+W \theta_{Z}\right) & =\theta_{X X}+\theta_{Z Z},
\end{aligned}
$$

where the Reynolds number, $\operatorname{Re}=\rho U_{0} h_{0} / \mu$, determines the ratio of inertial to viscous forces, and the Péclet number, $\mathrm{Pe}=\mathrm{RePr}$, characterizes the ratio of convection to diffusion, with the Prandtl number $\operatorname{Pr}=\mu / \rho \kappa$ measuring the ratio of momentum to thermal diffusivities.

The dimensionless boundary conditions at $Z=0$ are

$$
U=0, \quad W=0, \quad \theta=1 .
$$

At $Z=H$, the dimensionless forms of normal and tangential stresses balance, the energy balance, and the kinematic boundary conditions are

$$
\begin{aligned}
-P+\frac{2}{1+H_{X}^{2}}\left[U_{X}\left(H_{X}^{2}-1\right)-H_{X}\left(U_{Z}+W_{X}\right)\right] & =\frac{\mathrm{We}\left(1-\frac{\mathrm{Ma}}{\mathrm{We}} \theta\right)}{\left(1+H_{X}^{2}\right)^{3 / 2}} H_{X X}, \\
\frac{1}{\left(1+H_{X}^{2}\right)^{1 / 2}}\left[\left(U_{Z}+W_{X}\right)\left(1-H_{X}^{2}\right)-4 H_{X} U_{X}\right] & =-\mathrm{Ma}\left(\theta_{X}+H_{X} \theta_{Z}\right), \\
\frac{1}{\left(1+H_{X}^{2}\right)^{1 / 2}}\left(\theta_{Z}-H_{X} \theta_{X}\right) & =-\mathrm{Bi} \theta, \\
H_{\tau}+U H_{X}-W & =0,
\end{aligned}
$$

where the Weber number We $=\sigma_{0} / \mu U_{0}$ quantifies the ratio of capillary to viscous forces, the Marangoni number $\mathrm{Ma}=\sigma_{T}\left(T_{\mathrm{s}}-T_{\mathrm{g}}\right) / \mu U_{0}$ determines the ratio of thermocapillary to viscous 
TABLE I. Dimensionless parameters that determine the nonisothermal thin-film dynamics.

\begin{tabular}{lcl}
\hline \hline Parameter & Formula & \multicolumn{1}{c}{ Physical meaning } \\
\hline $\mathrm{Ma}$ & $\sigma_{T}\left(T_{\mathrm{s}}-T_{\mathrm{g}}\right) / \mu U_{0}$ & Ratio of thermocapillary to viscous forces \\
$\mathrm{Bi}$ & $h_{g} h_{0} / \lambda$ & Ratio of the conduction resistance of the liquid film \\
& & to the interfacial resistance \\
$\mathrm{Re}$ & $\rho U_{0} h_{0} / \mu$ & Ratio of inertial to viscous forces \\
$\mathrm{We}$ & $\sigma_{0} / \mu U_{0}$ & Ratio of capillary to viscous forces \\
\hline \hline
\end{tabular}

forces, with $\mathrm{Ma}>0$ corresponding to the heated substrate and $\mathrm{Ma}<0$ corresponding to the cooled substrate, and the Biot number $\mathrm{Bi}=h_{g} h_{0} / \lambda$ indicates the ratio of the conduction resistance of the liquid film to the interfacial resistance. These parameters are summarized in Table I.

\section{Long-wave approximation}

Using the long-wave theory and following the work of Kalliadasis et al. [30], we assume that the temporal and spatial variations of the interfacial slope are very slow and thus introduce a corresponding film parameter, $\epsilon \sim \partial_{\tau} \sim \partial_{X} \ll 1$. Based on this assumption, we introduce the following transformations:

$$
\left(\partial_{\tau}, \partial_{X}\right) \rightarrow \epsilon\left(\partial_{\tau}, \partial_{X}\right), \quad \partial_{X X} \rightarrow \epsilon^{2} \partial_{X X}, \quad W \rightarrow \epsilon W .
$$

After substituting Eq. (11) into Eqs. (8)-(10), the governing equations along with the associated boundary conditions become

$$
\begin{aligned}
U_{X}+W_{Z} & =0, \\
\epsilon \operatorname{Re}\left(U_{\tau}+U U_{X}+W U_{Z}\right) & =-\epsilon P_{X}+\epsilon^{2} U_{X X}+U_{Z Z}+1, \\
\epsilon^{2} \operatorname{Re}\left(W_{\tau}+U W_{X}+W W_{Z}\right) & =-P_{Z}+\epsilon^{3} W_{X X}+\epsilon W_{Z Z}+\cot \alpha, \\
\epsilon \operatorname{Pe}\left(\theta_{\tau}+U \theta_{X}+W \theta_{Z}\right) & =\epsilon^{2} \theta_{X X}+\theta_{Z Z} .
\end{aligned}
$$

At $Z=0$,

$$
U=0, \quad W=0, \quad \theta=1,
$$

and at $Z=H$,

$$
\begin{aligned}
& P=-2 \epsilon H_{X}\left(U_{Z}+\epsilon^{2} W_{X}\right)+2 \epsilon W_{Z}-\epsilon^{2} \mathrm{We}\left(1-\frac{\mathrm{Ma}}{\mathrm{We}} \theta\right) H_{X X}, \\
& U_{Z}+\epsilon^{2} W_{X}-2 \epsilon^{2} U_{X} H_{X}+2 \epsilon^{2} W_{Z} H_{X}=-\epsilon \mathrm{Ma} \theta_{X}, \\
& \epsilon^{2} H_{X} \theta_{X}-\theta_{Z}-\operatorname{Bi} \theta=0, \\
& H_{\tau}+U H_{X}-W=0,
\end{aligned}
$$

where all the nonlinear terms of $H_{X}^{2}$ are neglected, as we only consider the first-order long-wave model in this work. To simplify, the convective heat transfer term is neglected in the energy Eq. (12d) by assuming $\epsilon \mathrm{Pe} \ll 1$. In addition, as we consider a small temperature difference, $T_{\mathrm{s}}-T_{\mathrm{g}} \sim O(10) \mathrm{K}$, the term of $\frac{\mathrm{Ma}}{\mathrm{We}} \ll 1$ in Eq. (14a), and it is also neglected. For instance, taking 200 -cSt silicone oil at room temperature as an example [28], its dynamic viscosity $\mu \approx 0.2 \mathrm{~Pa} \mathrm{~s}$, surface tension $\sigma_{0}=2.1 \times 10^{-2} \mathrm{~N} / \mathrm{m}$, and surface-tension parameter $\sigma_{T}=6.8 \times 10^{-5} \mathrm{~N} / \mathrm{mK}$. Given by the temperature difference of $T_{\mathrm{s}}-T_{\mathrm{g}} \approx 10 \mathrm{~K}$ and the characteristic velocity $U_{0}$ of $10^{-2} \sim$ $10^{-3} \mathrm{~m} / \mathrm{s}$, we can estimate that $\mathrm{Ma} \sim O\left(10^{-1} \sim 1\right)$, We $\sim O\left(10 \sim 10^{2}\right)$, and $\frac{\mathrm{Ma}}{\mathrm{We}} \sim O\left(10^{-2}\right)$. 
To get the asymptotic solutions of Eqs. (12)-(14), we further expand all variables in power series of $\epsilon$ in the form of

$$
[U, V, P, \theta]=\left[U^{(0)}, V^{(0)}, P^{(0)}, \theta^{(0)}\right]+\epsilon\left[U^{(1)}, V^{(1)}, P^{(1)}, \theta^{(1)}\right]+O\left(\epsilon^{2}\right),
$$

where $\left[U^{(0)}, V^{(0)}, P^{(0)}, \theta^{(0)}\right]$ and $\left[U^{(1)}, V^{(1)}, P^{(1)}, \theta^{(1)}\right]$ are the leading-order and the first-order solutions, respectively. It should be noted that we consider up to the first-order $O(\epsilon)$ flow dynamics, and higher-order terms $O\left(\epsilon^{2}\right)$ in Eq. (15) will be neglected in the following analysis [32].

After inserting Eq. (15) into Eqs. (12)-(14) and making some calculations, we obtain the leadingorder solution $U^{(0)}, V^{(0)}, P^{(0)}$, and $\theta^{(0)}$ :

$$
\begin{aligned}
U^{(0)} & =-\frac{1}{2} Z^{2}+H Z, \\
W^{(0)} & =-\frac{1}{2} H_{X} Z^{2}, \\
P^{(0)} & =\cot \alpha(Z-H)-\epsilon^{2} \mathrm{We} H_{X X}, \\
\theta^{(0)} & =1-\frac{\mathrm{Bi} Z}{1+\mathrm{Bi} Z},
\end{aligned}
$$

where the leading-order interfacial temperature is therefore obtained as $\theta^{\mathrm{i},(0)}=\left.\theta^{(0)}\right|_{Z=H}=\frac{1}{1+\mathrm{Bi} H}$. The first-order solutions $U^{(1)}$ and $P^{(1)}$ are calculated as

$$
\begin{aligned}
U^{(1)}= & \left(-\cot \alpha H_{X}-\epsilon^{2} \mathrm{We} H_{X X X}\right)\left(\frac{Z^{2}}{2}-H Z\right)+\frac{\operatorname{MaBi} H_{X}}{(1+\operatorname{Bi} H)^{2}} Z \\
& +\operatorname{Re}\left[\left(\frac{Z^{4}}{24}-\frac{H^{3} Z}{6}\right) H H_{X}+\left(\frac{Z^{3}}{6}-\frac{H^{2} Z}{2}\right) H_{\tau}\right], \\
P^{(1)}= & -(Z-H) H_{X}-2 H H_{X} .
\end{aligned}
$$

The detailed calculations of the above leading-order and first-order solutions are illustrated in Appendix A.

We then substitute the streamwise velocity profile, $U=U^{(0)}+\epsilon U^{(1)}$, into the kinematic condition Eq. (14d), which is now written in its mass conservation form,

and we can obtain

$$
H_{\tau}+\partial_{X} \int_{0}^{H} U d Z=0
$$

$H_{\tau}+H^{2} H_{X}+\epsilon\left[\frac{H^{3}}{3}\left(\cot \alpha H_{X}+\epsilon^{2} \mathrm{We} H_{X X X}\right)+\frac{\operatorname{MaBi} H^{2} H_{X}}{2(1+\mathrm{Bi} H)^{2}}-\operatorname{Re}\left(\frac{3 H^{6} H_{X}}{40}+\frac{5 H^{4}}{24} H_{\tau}\right)\right]_{X}=0$.

To eliminate the term of $H_{\tau}$ in Eq. (19), we apply $H_{\tau}=-H^{2} H_{X}+O(\epsilon)$, and in addition, to compare the results of linear stability analysis from the long-wave model with those from the linearized NS equation, we further make the following transformations:

$$
\epsilon\left(\partial_{\tau}, \partial_{X}\right) \rightarrow\left(\partial_{\tau}, \partial_{X}\right)
$$

To this end, we eventually obtain the first-order long-wave model describing the dynamics of a nonisothermal liquid film flowing under an inclined substrate as follows:

$$
H_{\tau}+\underbrace{H^{2} H_{X}}_{\mathrm{I}}+[\underbrace{\frac{1}{3} \cot \alpha H^{3} H_{X}}_{\mathrm{II}}+\underbrace{\frac{1}{3} \mathrm{We} H^{3} H_{X X X}}_{\text {III }}+\underbrace{\frac{\mathrm{MaBi}}{2(1+\mathrm{Bi} H)^{2}} H^{2} H_{X}}_{\text {IV }}+\underbrace{\frac{2}{15} \operatorname{Re} H^{6} H_{X}}_{\mathrm{V}}]_{X}=0,
$$


where term I represents the convective term due to mean flow under the gravity component parallel to the substrate, term II denotes the gravity component perpendicular to the substrate, term III corresponds to the surface-tension effect, term IV accounts for the thermocapillary stress due to the interfacial temperature gradient, and term $\mathrm{V}$ is the fluid inertia. The expressions and physical meanings of dimensionless parameters that appear in Eq. (21) are listed in Table I. It should be noted that the prefactor of term $\mathrm{V}$ involving fluid inertia is $2 / 15$, rather than $2 / 5$ in Ref. [30]. This mismatch results from different characteristic length and timescales, and the definition of the Reynolds number. For the extreme limit of $\mathrm{Ma} \rightarrow 0$, the thermocapillary stress term in Eq. (21) vanishes and the first-order model presented in the work of Scheid et al. [29] can be recovered. By further considering the limit of negligible inertia $(\operatorname{Re} \rightarrow 0)$ and representing Eq. (21) with dimensional terms, the lubrication model derived by Brun et al. [5] can also be recovered. In this work we concentrate on the effects of the thermocapillarity Ma and the inclination angle $\alpha$ of the substrate on the RT instability, and therefore we consider a wide range of these two parameters, i.e., Ma $\in[-5,5]$ and $\alpha \in(0, \pi / 2)$. Furthermore, to avoid the finite-time blowup during the numerical simulations of Eq. (21) [33], a small Reynolds number, $\operatorname{Re} \in$ [0,2], is considered and the classical Kapitza instability [34] is not taken into consideration.

\section{LINEAR STABILITY ANALYSIS}

The linear stability of the base state is examined by decomposing the film thickness $H$ into its spatial base solution $\bar{H}=1$ with a small space-dependent perturbation $H^{\prime}$, which reads

$$
H=1+H^{\prime} \text {. }
$$

After injecting Eq. (22) into Eq. (21) and collecting all the linearized terms of $H^{\prime}$, we obtain the following linear equation for the perturbation:

$$
H_{\tau}^{\prime}+H_{X}^{\prime}+\left[\frac{1}{3} \cot \alpha H_{X}^{\prime}+\frac{1}{3} \mathrm{We} H_{X X X}^{\prime}+\frac{\mathrm{MaBi}}{2(1+\mathrm{Bi})^{2}} H_{X}^{\prime}+\frac{2}{15} \mathrm{Re} H_{X}^{\prime}\right]_{X}=0
$$

We perform a standard normal-mode analysis by employing

$$
H^{\prime}=\hat{H} \exp (i k X+\omega \tau),
$$

where $i=\sqrt{-1}$, and $k, \omega$, and $\hat{H}$ denote the complex wave number, complex wave frequency, and amplitude of the perturbation, respectively. By applying this normal-mode decomposition to Eq. (23) and linearizing the system based on $\hat{H} \ll 1$, we derive the general dispersion relation $\omega(k)$

$$
\omega=-i k+\frac{1}{3} k^{2} \cot \alpha-\frac{1}{3} \mathrm{We} k^{4}+\frac{\mathrm{MaBi}}{2(1+\mathrm{Bi})^{2}} k^{2}+\frac{2}{15} \mathrm{Re} k^{2} .
$$

The imaginary part $\omega_{i}$ and real part $\omega_{r}$ of $\omega$ quantify the advection of the perturbed wave and the temporal growth of the perturbation, respectively.

\section{A. Temporal stability analysis}

Based on Eq. (25), we obtain

$$
\omega_{r}=\frac{1}{3} k^{2} \cot \alpha-\frac{1}{3} \mathrm{We} k^{4}+\frac{\mathrm{MaBi}}{2(1+\mathrm{Bi})^{2}} k^{2}+\frac{2}{15} \operatorname{Re} k^{2} .
$$

Note that the flow is temporally unstable, marginally stable, and stable when $\omega_{r}>0, \omega_{r}=0$, and $\omega_{r}<0$, respectively. By setting $\omega_{r}=0$, we derive the cutoff wave number $k_{c}$ that divides the stable and unstable regions,

$$
k_{c}=\sqrt{\frac{1}{\mathrm{We}}\left(\cot \alpha+\mathrm{Ma}^{*}+\frac{2}{5} \mathrm{Re}\right)},
$$


(a)

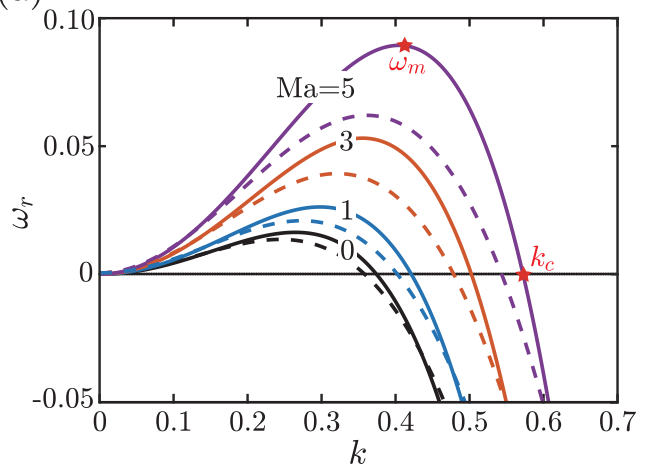

(b)

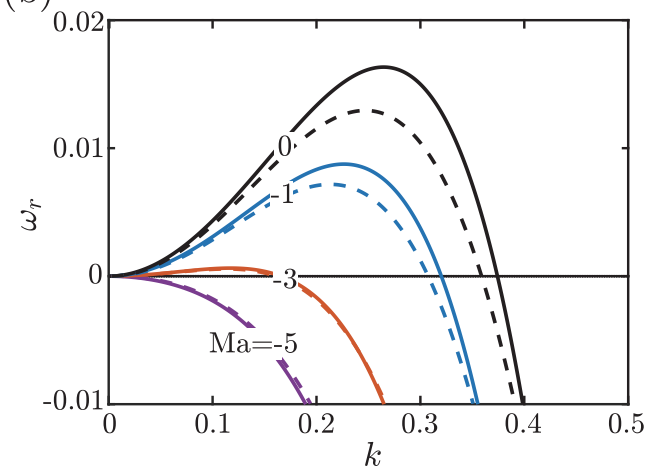

FIG. 2. Temporal growth rates $\omega_{r}$ vs wave number $k$ for a film under: (a) a heated substrate with $M a=1$, 3 , and 5; (b) a cooled substrate with $\mathrm{Ma}=-1,-3$, and -5 . The cutoff wave number $k_{c}$ and maximal growth rate $\omega_{m}$ when $\mathrm{Ma}=5$ are marked by red stars in (a). The results for an isothermal film $(\mathrm{Ma}=0)$ are also depicted for reference. Solid and dashed lines correspond to the results from the linearized long-wave model and the linearized NS equation, respectively. Here, $\alpha=\pi / 4$, We $=10, \mathrm{Bi}=1$, and $\operatorname{Re}=1$.

where $\mathrm{Ma}^{*}=\frac{3 \mathrm{MaBi}}{2(1+\mathrm{Bi})^{2}}$ is introduced as a composite Marangoni number. The wave number of the most unstable mode $k_{m}$ can be yielded by letting the derivative $d \omega_{r} / d k=0$ :

$$
k_{m}=\sqrt{\frac{1}{\mathrm{We}}\left(\frac{1}{2} \cot \alpha+\frac{3}{2} \mathrm{Ma}^{*}+\frac{1}{5} \mathrm{Re}\right)} .
$$

From Eqs. (26) and (28), we can clearly see that in the absence of substrate heating or cooling, i.e., $\mathrm{Ma}=0$ or equivalently, $\mathrm{Ma}^{*}=0, k_{c}$ and $k_{m}$ are strictly positive, indicating the flow is always temporally unstable under an inclined plane $(0<\alpha<\pi / 2)$. This unconditional instability is due to the amplification of long waves by the gravity component perpendicular to the substrate, namely, $g \cos \alpha$, triggering the well-known RT instability [1,2].

The temporal growth rates $\omega_{r}$ as a function of wave number $k$ for a heated and a cooled substrate when $\alpha=\pi / 4$ are shown in Figs. 2(a) and 2(b), respectively. The isothermal cases with Ma $=0$ are also illustrated in Fig. 2 for reference. When the film is heated by the substrate, i.e., $\mathrm{Ma}>0$, the thermocapillary stress destabilizes the system compared to the isothermal case, as indicated by the increased cutoff wave number $k_{c}$ and maximal growth rate $\omega_{m}$ [Fig. 2(a)]. However, when the film is cooled by the substrate, i.e., $\mathrm{Ma}<0$, a totally different phenomenon can be observed, where the thermocapillary stress stabilizes the film, as reflected by the decreased $k_{c}$ and $\omega_{m}$ [Fig. 2(b)]. In particular, strong cooling such as $\mathrm{Ma}=-5$ can totally suppress the temporal RT instability, in which all eigenvalues are in the stable complex half-plane $\left[\omega_{r}<0\right.$, see Fig. 2(b)]. The temporal stability analysis of the long-wave evolution equation is further compared with the numerical solutions of the linearized NS equation (see the details in Appendix B). We observe that the cutoff wave number $k_{c}$ and the most unstable mode $k_{m}$ obtained from the long-wave theory qualitatively agree with those from the linearized NS equation [see dashed lines in Figs. 2(a) and Fig. 2(b)]. This agreement holds evidently for a cooled substrate [Fig. 2(b)], or when the capillary effect indicated by We is strong, as shown in Fig. 9 in Appendix B. The discrepancy in dispersion curves between the long-wave model and the linearized NS equation can be improved by employing the method of weighted residuals [35,36], as also discussed recently by Scheid et al. [29]. In this work, however, we simply use the first-order long-wave evolution equation to capture the main flow characteristics.

We further show in Figs. 3(a) and 3(b), respectively, the marginal stability curves dividing the linearly stable and unstable regions in the parameter space $\mathrm{Ma}-k_{c}$ for a heated and a cooled substrate, where $\alpha=\pi / 12, \pi / 6$, and $\pi / 3$. For a given Ma, the linearly unstable region shrinks with 
(a)

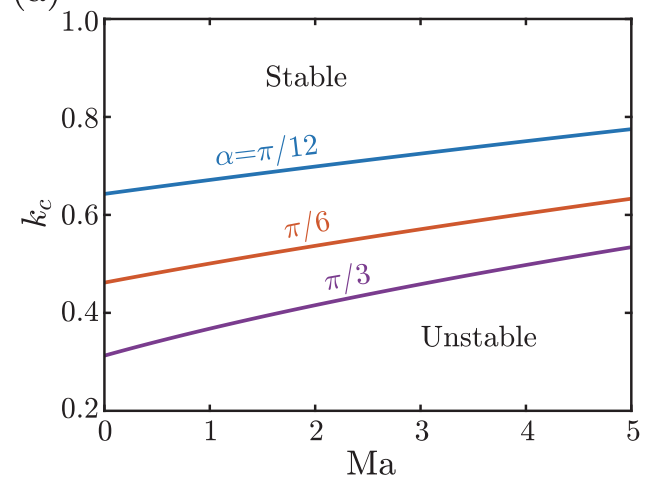

(b)

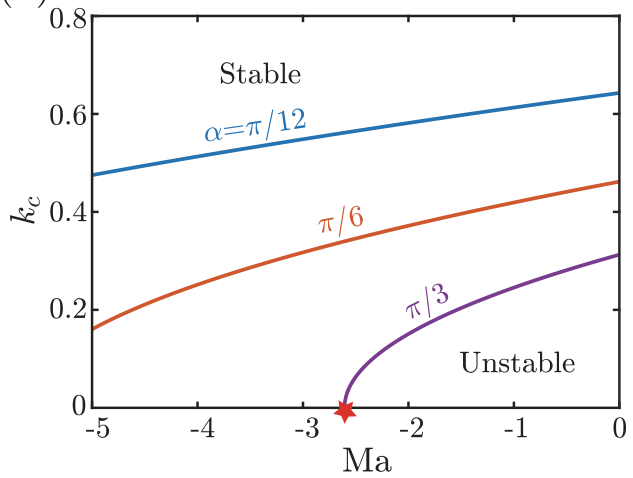

FIG. 3. Marginal stability curves demarcating the stable and unstable regions in the $\mathrm{Ma}-k_{c}$ plane when $\alpha=\pi / 12, \pi / 6$, and $\pi / 3$ for a film under (a) a heated substrate $(\mathrm{Ma}>0)$ and $(\mathrm{b})$ a cooled substrate $(\mathrm{Ma}<0)$. In (b), the cutoff Marangoni number for the case of $\alpha=\pi / 3$ is also marked (red star). Here, We $=10, \mathrm{Bi}=1$, and $\operatorname{Re}=1$.

increasing $\alpha$, indicating that a larger inclination angle stabilizes the film more strongly. Notably, for a cooled substrate, when the composite Marangoni number $\mathrm{Ma}^{*}$ is below a critical value $\mathrm{Ma}_{1}^{*}$,

$$
\mathrm{Ma}_{1}^{*}=-\left(\cot \alpha+\frac{2}{5} \mathrm{Re}\right),
$$

and the temporal RT instability is completely inhibited [see Eq. (27)]. In other words, Ma* demarcates the temporally stable and unstable regions. This result is consistent with an earlier study [26] on the thermocapillary liquid film suspending under a cooled horizontal substrate $(\alpha=0)$, which showed that the RT instability can be suppressed by a sufficiently large vertical temperature difference. The physical mechanism underlying the thermocapillary effect on the flow dynamics can be qualitatively elaborated as follows: If an externally random perturbation with a small amplitude is imposed on the liquid-air interface, thick parts (crests) and thin parts (troughs) on the interface can be formed. As mentioned before, the surface tension decreases with temperature. Therefore, when the film is heated by the substrate $\left(T_{\mathrm{s}}>T_{\mathrm{g}}\right)$, the liquid at the trough is hotter than that in the crest and thus has a lower surface tension, giving rise to a local surface-tension gradient. This surface-tension gradient will pull the liquid from the hot trough into the cold crest. This means that the resulting thermocapillary stress reinforces the gravitational RT mechanism to further destabilize the flow. The reverse argument holds true when the film is cooled by the substrate $\left(T_{\mathrm{s}}<T_{\mathrm{g}}\right)$, where the liquid is pulled from the crest into the trough and the thermocapillary stress competes with the gravitational RT mechanism, stabilizing the flow.

For a liquid film much thinner than the capillary length, the RT instability is varied from a horizontal substrate to a tilted counterpart [5,29]. The tangential component of the gravity induces a flow that can advect the perturbation so that the instability is partially changed for a small inclination angle but might be entirely annihilated for a large inclination angle. The transition between these two regimes for the isothermal liquid film has been rationalized using the absolute and convective instability analysis, which well accounts for whether drops can form or not [5,29]. However, the thermocapillary effect on the spatiotemporal stability of a nonisothermal liquid film remains unexplored. Compared to existing studies on isothermal films [5,29], the addition of a positive or negative thermocapillary contribution term in the evolution Eq. (21) and dispersion relation Eq. (25) might largely modify the competition between instability growth and its advection and thus change the absolute/convective transition. The temporal stability analysis presented in Sec. III A determines whether the flow is temporally stable or not but fails to provide information on the nature of the unstable flow, i.e., the absolutely or the convectively unstable. Therefore, in the following section 

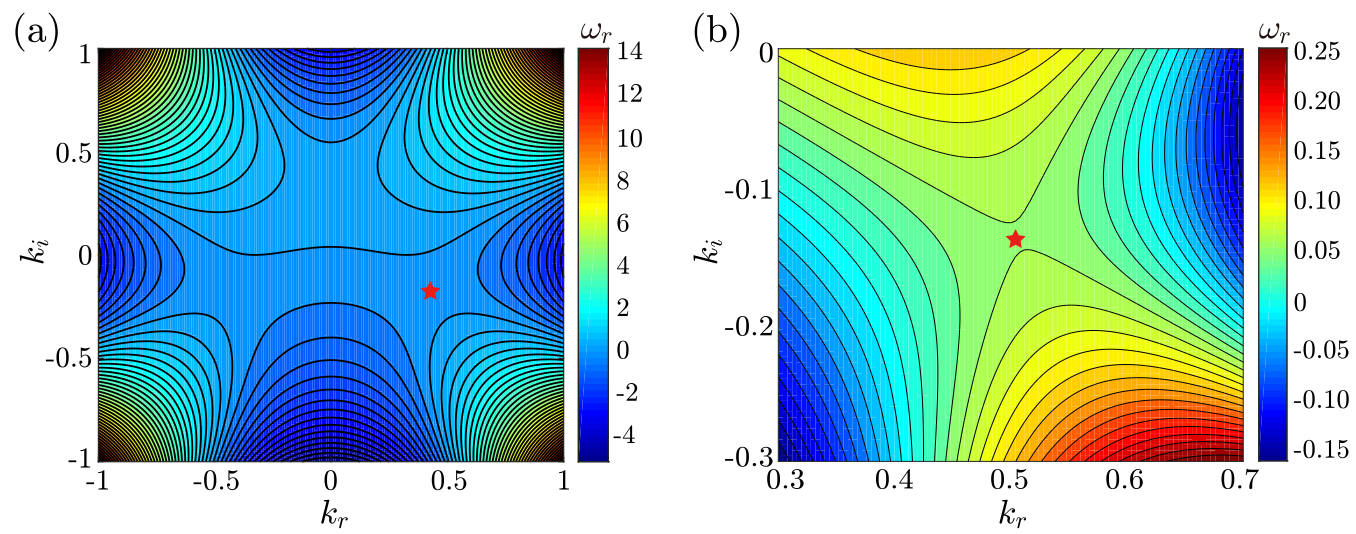

FIG. 4. Contour maps of $\omega_{r}$ in the complex plane $\left(k_{r}, k_{i}\right)$. (a) An isothermal film $(\mathrm{Ma}=0)$ where a saddle point locates at $\left(k_{r}, k_{i}\right)=(0.44,-0.17)$ (red star) with real growth rate $\omega_{r}=-0.07$. (b) A heated film (Ma $=$ 5) where a saddle point $\left(k_{r}, k_{i}\right)=(0.51,-0.14)$ (red star) locates in the fourth quadrant with the real growth rate $\omega_{r}=0.06$. Here, $\alpha=\pi / 6, \mathrm{We}=10, \mathrm{Bi}=1$, and $\mathrm{Re}=1$.

we examine the thermocapillary effect on the absolute and convective instability of the temporally unstable film via a spatiotemporal analysis.

\section{B. Spatiotemporal stability analysis}

The concept of convective and absolute stability is firstly developed in plasma instabilities [37,38] and was recently extended to the problems of hydrodynamics [39]. The convective/absolute stability property of a given flow is determined by the long-time behavior of an impulsive response by a localized excitation. If the amplified perturbation propagates both upstream and downstream of the source and invades the entire spatial domain, the flow is absolutely unstable; however, if the amplified perturbation moves away from the source while it grows in amplitude but eventually leaves the spatial domain, the flow is convectively unstable. The type of convective/absolute instability can be distinguished by the most amplified wave of zero group velocity, i.e., the temporal growth rate at a saddle point in the complex plane $\left(k_{r}, k_{i}\right)$. The saddle point should be from the collision of two spatial branches coming from the opposite sides of the horizontal axis [39].

To determine the convective and absolute instability, we first obtain the implicit expression of saddle points in the complex $k$ plane by setting $\partial \omega /\left.\partial k\right|_{k=k_{0}}=0$, namely,

$$
-i+\frac{2}{3} \cot \alpha k-\frac{4}{3} \mathrm{We} k^{3}+\frac{\mathrm{MaBi}}{(1+\mathrm{Bi})^{2}} k+\frac{4}{15} \mathrm{Re} k=0,
$$

where $k_{0}$ is the saddle point with the corresponding absolute frequency $\omega_{0}=\left.\omega\right|_{k=k_{0}}$. At the saddle point, the real part of $\omega_{0}, \omega_{0, r}$ marks the boundary between an absolutely unstable flow and a convectively unstable flow: the flow is absolutely unstable when $\omega_{0, r}>0$ and convectively unstable when $\omega_{0, r}<0$.

A typical contour plot of the temporal growth rate $\omega_{r}$ for an isothermal liquid film with $\alpha=\pi / 6$ in the complex $k$ plane, $\left(k_{r}, k_{i}\right)$, is shown in Fig. 4(a). We can identify three saddle points in this complex plane, including one in the upper half-plane and another two in the lower half-plane. However, the upper saddle point does not satisfy the Briggs-Bers collision criterion [37,38]. Although both saddle points in the lower half-plane are applicable, we here adopt the saddle point in the fourth quadrant to determine the absolute/convective instability [40]. For this isothermal film, at the saddle point $k=0.44-0.17 i$, the real part of the temporal frequency $\omega_{0, r}=-0.07<0$, indicating the flow is convectively unstable [Fig. 4(a)]. However, when the substrate is heated, for instance, $\mathrm{Ma}=5$, at its saddle point $k=0.51-0.14 i$, the real part of the temporal frequency is positive 
(a)

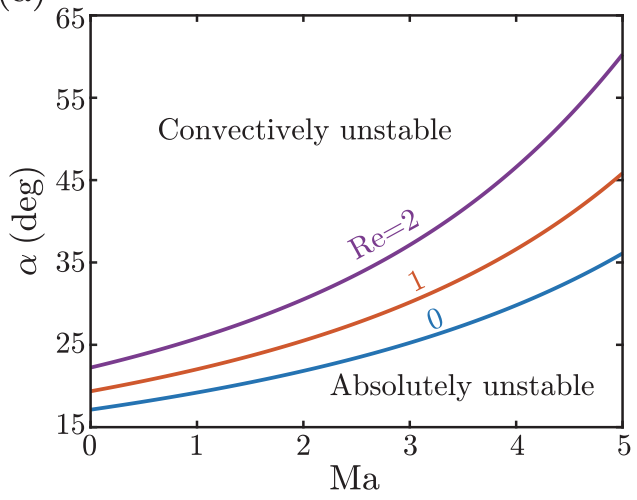

(b)

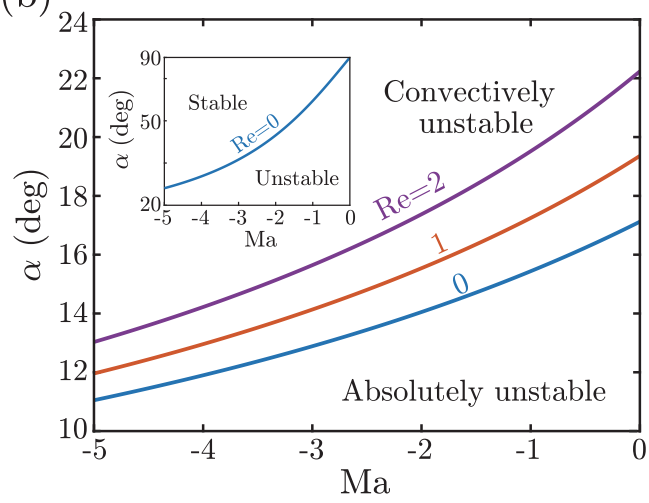

FIG. 5. Marginal stability curves in the $\mathrm{Ma}-\alpha$ plane when $\mathrm{Re}=0,1$, and 2 for a film under (a) a heated substrate and (b) a cooled substrate. The inset of (b) shows the marginal stability curve demarcating the (temporally) stable and unstable regions in the $\mathrm{Ma}-\alpha$ plane when $\mathrm{Re}=0 . \mathrm{Here}, \mathrm{We}=10$ and $\mathrm{Bi}=1$.

$\left[\omega_{0, r}=0.06\right.$, see Fig. 4(b)], indicating that the flow becomes absolutely unstable. For a cooled substrate such as $\mathrm{Ma}=-5$ with the same angle $\alpha=\pi / 6$, at its saddle point $k=0.37-0.21 i$ the real part of the temporal frequency is negative $\left(\omega_{0, r}=-0.15\right)$, indicating a convectively unstable flow. In this case the absolute value of $\omega_{0, r}$ becomes larger, implying that the flow behaves much more convectively than the isothermal case. These preliminary findings suggest that the thermocapillary stress can largely modify the transition between the absolute and convective instability for a prescribed inclination angle.

To better understand how the thermocapillary stress affects the transition boundary between the absolute and convective instability, we refer to the marginal curve that divides these two unstable regions. First we multiply the dispersion relation Eq. (25) by $i$ to obtain the following rescaled form:

$$
\tilde{\omega}=\tilde{k}+\frac{i \tilde{k}^{2}}{3}\left(b-\tilde{k}^{2}\right)
$$

where

$$
i \omega=\mathrm{We}^{-\frac{1}{3}} \tilde{\omega}, \quad k=\mathrm{We}^{-\frac{1}{3}} \tilde{k}, \quad b=\left[\cot \alpha+\frac{3 \mathrm{MaBi}}{2(1+\mathrm{Bi})^{2}}+\frac{2}{5} \mathrm{Re}\right] \mathrm{We}^{-\frac{1}{3}} .
$$

This form has also been similarly derived by Duprat et al. [41], Ding et al. [42], and Khanum and Tiwari [43] for the Rayleigh-Plateau instability in liquid films falling down a cylindrical substrate. By equating the absolute growth rate $\tilde{\omega}_{r}$ at the saddle point in Eq. (31) with zero, we derive the marginal curves characterized by the critical value, $b_{c}=\left[\frac{9}{4}(-17+7 \sqrt{7})\right]^{1 / 3} \simeq 1.507$ [41]. The sign of $b_{c}$ determines the nature of the instability, which is absolute when $b>b_{c}$ but convective when $b<b_{c}$. In other words, the absolute and convective instability region can be distinguished by another critical value $\mathrm{Ma}_{2}^{*}$,

$$
\mathrm{Ma}_{2}^{*}=\left[\frac{9}{4}(-17+7 \sqrt{7}) \mathrm{We}\right]^{\frac{1}{3}}-\left(\cot \alpha+\frac{2}{5} \mathrm{Re}\right),
$$

where the flow is absolutely unstable when $\mathrm{Ma}^{*}>\mathrm{Ma}_{2}^{*}$ and convectively unstable when $\mathrm{Ma}_{1}^{*}<$ $\mathrm{Ma}^{*}<\mathrm{Ma}_{2}^{*}$. Interestingly, we note that for arbitrary combinations of $\mathrm{Re}$ and $\cot \alpha(0<\alpha<\pi / 2$ of the suspending film in this work), $\mathrm{Ma}_{2}^{*}$ is less than $\left[\frac{9}{4}(-17+7 \sqrt{7}) \mathrm{We}\right]^{\frac{1}{3}}$ [see Eq. (32)]. This means an absolutely unstable flow always emerges when $\mathrm{Ma}^{*}>\left[\frac{9}{4}(-17+7 \sqrt{7}) \mathrm{We}\right]^{\frac{1}{3}}$.

The marginal stability curves in the parameter space $(\mathrm{Ma}, \alpha)$ for $\mathrm{Ma}>0$ and $\mathrm{Ma}<0$ with various Re are plotted in Figs. 5(a) and 5(b), respectively. When Ma $>0$, increasing Ma can switch 
the flow from being convectively unstable to being absolutely unstable at a given $\alpha$. This transition indicates that the heating-induced thermocapillary stress can promote the absolute instability for an enhanced formation of suspending droplets. Furthermore, by increasing Re from 0 to 2, the absolutely unstable regime expands counterclockwise in the $\mathrm{Ma}-\alpha$ plane, suggesting that the fluid inertia can accelerate the absolute instability. When $\mathrm{Ma}<0$, a totally opposite tendency is revealed. As shown in Fig. 5(b), increasing $|\mathrm{Ma}|$ allows the flow to transit from the absolute instability to the convective instability and finally to temporal stability. This transition suggests that when the Marangoni number is above a threshold, the cooling-induced thermocapillary stress suppresses the absolute instability and hence impedes the droplet formation. In this Ma $-\alpha$ plane [Fig. 5(b)], with increasing $\mathrm{Re}$ from 0 to 2 , the convectively unstable region shrinks and the absolutely unstable region expands counterclockwise as well. To this end, for either $\mathrm{Ma}>0$ or $\mathrm{Ma}<0$, the flow is prone to be absolutely unstable when strong fluid inertia (higher $\mathrm{Re}$ ) is present.

To apply the derived stability thresholds, i.e., Eqs. (29) and (32), into real systems, we again consider the 200-cSt silicone oil as the working fluid. Its physical properties at room temperature $T_{\mathrm{g}}$ of $20^{\circ} \mathrm{C}$ are $\rho=0.969 \mathrm{~g} / \mathrm{cm}^{3}, \mu=0.2 \mathrm{~Pa} \mathrm{~s}, \sigma_{0}=2.1 \times 10^{-2} \mathrm{~N} / \mathrm{m}, \sigma_{T}=6.8 \times 10^{-5} \mathrm{~N} / \mathrm{mK}$, $\lambda=0.155 \mathrm{~W} / \mathrm{mK}$, and $h_{\mathrm{g}} \approx 250 \mathrm{~W} / \mathrm{m}^{2} \mathrm{~K}[27,28,30]$. If the film thickness $h_{0}=0.2 \mathrm{~mm}$ and the inclination angle of substrate $\alpha=\pi / 4$, the dimensionless parameters Bi, Re, and We in Table I are estimated to be $0.3,0.001$, and 80 , respectively. Therefore, without applying wall heating/cooling $(\mathrm{Ma}=0), b=0.23<b_{c}$, leading to a convectively unstable film. To stabilize the film, the substrate temperature $T_{\mathrm{s}}$ should be maintained below $5.7^{\circ} \mathrm{C}$, according to Eq. (29); to trigger the absolutely unstable flow, $T_{\mathrm{s}}$ should be above $98^{\circ} \mathrm{C}$, calculated from Eq. (32).

\section{TRANSIENT NUMERICAL SIMULATIONS}

To further demonstrate how the thermocapillary stress affects the absolute and convective flow patterns, we need to numerically solve the full nonlinear evolution, Eq. (21). The simulations are carried out in a periodic domain with $X \in[0, L]$, where $L$ is the length of domain. The Fourier spectral method [44] is used for space discretization as

$$
H(X, \tau)=\sum_{m=-N / 2}^{N / 2} \hat{H}_{m}(\tau) \exp \left(i m \frac{2 \pi}{L} X\right),
$$

where $\hat{H}_{m}(\tau)$ and $N$ are the time-dependent Fourier coefficient and the number of Fourier modes, respectively. Gear's method [45] is adopted for time marching with a relative error below $10^{-8}$ of the solutions [46].

\section{A. Numerical solutions for the temporal evolution}

We first perform transient numerical simulations to solve Eq. (21) and examine the temporal stability analysis conducted in Sec. III A. A sinusoidal perturbation with the optimal wave number, i.e., the most unstable mode $k_{m}$ given by Eq. (28), is applied as the initial condition. The length of the computational domain is set as $L=2 \pi / k_{m}$, and the number of Fourier modes is $N=256$. The temporal growth $A$ of the perturbation is defined as [43]

$$
A=\ln \left[\frac{\|H(X, \tau)-\bar{H}\|_{2}}{\|H(X, 0)-\bar{H}\|_{2}}\right],
$$

where $\|(\cdot)\|_{2}$ and $\bar{H}=1$ represent the 2-norm and the base state, respectively. The temporal evolution of $A$ for four different Marangoni numbers, Ma $=-1,0,1$, and 3, are shown in Fig. 6(a). Similar to Sec. III A, other parameters are fixed: $\alpha=\pi / 4, \mathrm{We}=10, \mathrm{Bi}=1$, and $\mathrm{Re}=1$. Figure 6(a) indicates the exponential growth of the perturbation at the early stage and its nonlinear saturation at the later stage, as reflected by the temporal evolution of film thickness $H$. For instance, in the case of $\mathrm{Ma}=3$, at the early stage $(\tau<60)$, the amplitude of perturbation 
(a)

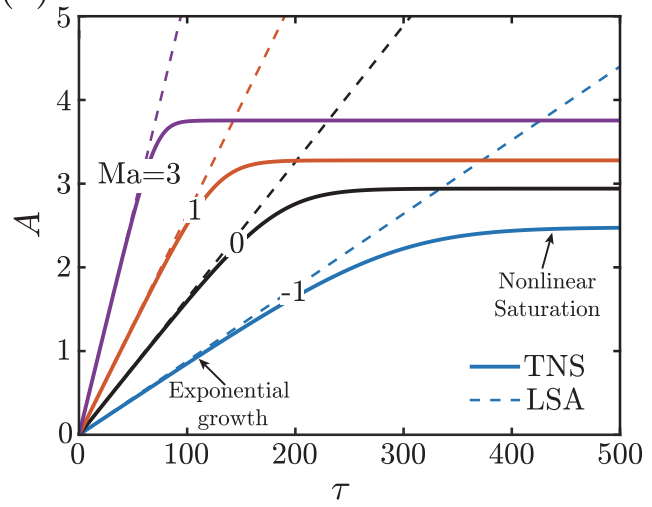

(b1)

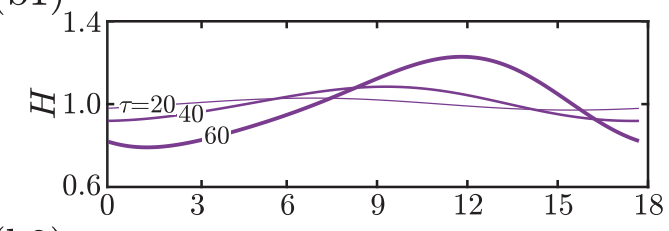

(b2)

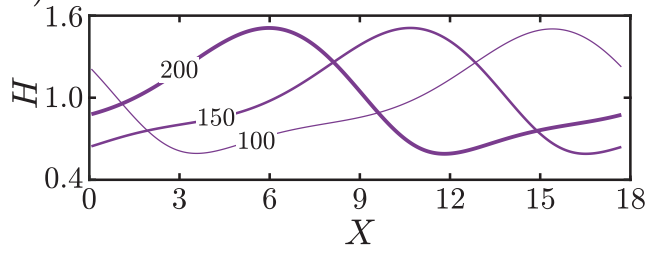

FIG. 6. (a) Temporal growth $A$ of the perturbations from the transient numerical solutions (TNS, solid lines) of the full evolution equation and that based on linear stability analysis (LSA, dashed lines) when Ma $=-1$, 0,1 , and 3. (b1), (b2) The temporal evolution of the initial sinusoidal perturbations at the early, exponentially growing stage (b1) and the later, nonlinear saturation stage (b2) with Ma $=3$. Here, $\alpha=\pi / 4$, We $=10$, $\mathrm{Bi}=1$, and $\mathrm{Re}=1$.

wave rapidly grows with time [see Fig. 6(b1)], and at a later interval $(\tau>100)$, the amplitude reaches a plateau due to nonlinear saturation [Fig. 6(b2)]. As we here choose perturbations with the optimal mode as the initial condition, the slop in the regime of exponential growth represents the maximal temporal growth rate. For comparison, we also depict the growth rates from the linear stability analysis as dashed lines in Fig. 6(a), which quantitatively agree with the numerical results.

\section{B. Numerical solutions for the spatiotemporal evolution}

We now proceed to performing transient numerical simulations to examine the spatiotemporal stability analysis conducted in Sec. III B. A localized impulse disturbance at $X=100$ is initially seeded into the flat film in the form of

$$
H=1+0.1 \exp \left[-\frac{1}{2}(X-100)^{2}\right] .
$$

Commonly, for a convective instability, both the leading $\left(V_{f}^{+}\right)$and receding $\left(V_{f}^{-}\right)$front velocities of the impulse response are positive, and therefore the instability will be convected away from the disturbed position. In contrast, an absolute instability is characterized with positive $V_{f}^{+}$and negative $V_{f}^{-}$, in which the entire domain will be contaminated by the initial disturbance. This concept can be well illustrated by observing the spatiotemporal evolution of the wave packet emerging from the initial localized excitation [5].

We here consider two representative scenarios: (i) $\mathrm{Ma}=0$ and $\mathrm{Ma}=5$ with a large angle $\alpha=\pi / 6$, and (ii) $\mathrm{Ma}=0$ and $\mathrm{Ma}=-5$ with a small angle $\alpha=\pi / 12$. Other parameters are fixed: $\mathrm{We}=10, \mathrm{Bi}=1$, and $\mathrm{Re}=1$. As inferred by the spatiotemporal stability analysis in Sec. III B, the following applies: (i) When $\alpha=\pi / 6$, the isothermal film is linearly unstable with a convective nature; however, in the presence of strong substrate heating $(\mathrm{Ma}=5)$, the flow will become absolutely unstable [see Fig. 5(a)]. (ii) When $\alpha=\pi / 12$, the isothermal flow is also linearly unstable but with an absolute nature; however, in the presence of strong substrate cooling $(\mathrm{Ma}=-5)$, the flow will be convectively unstable [Fig. 5(b)]. During the simulations, an extended domain length $L=400$ and an increased number $N=512$ of Fourier modes are applied to accurately capture the spatial evolution of the imposed disturbance. Furthermore, to avoid the fouling of disturbance at the upstream, especially for the situation of convectively unstable flows, we 
(a1)

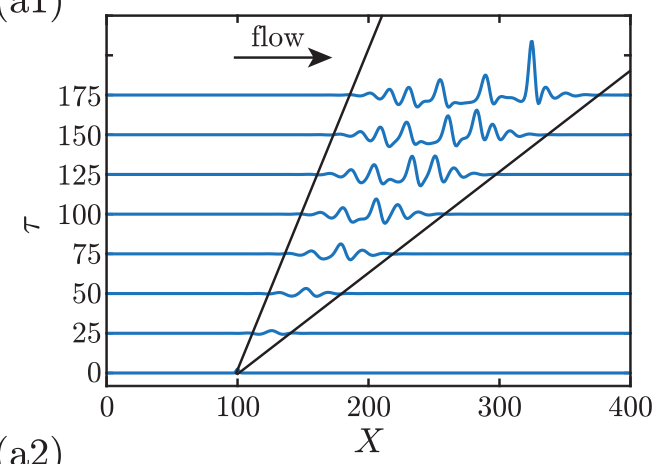

(a2)

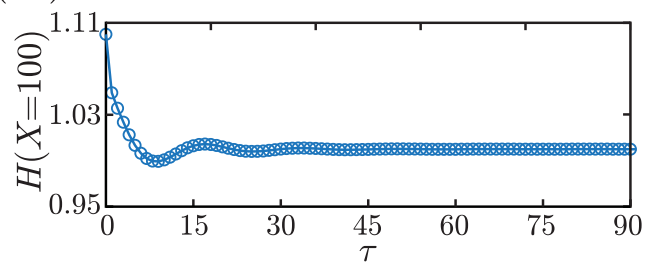

(b1)

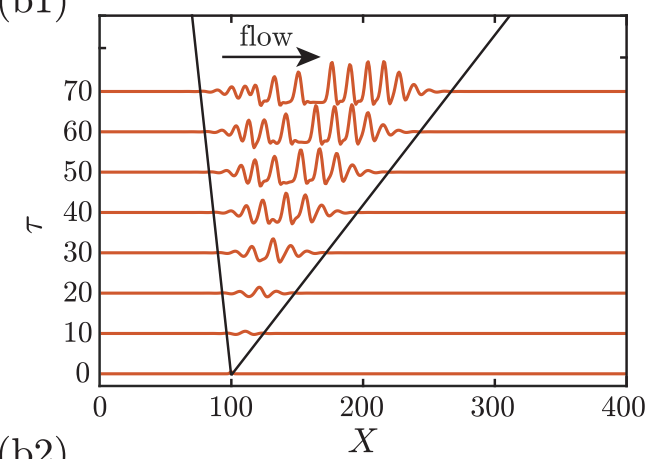

(b2)

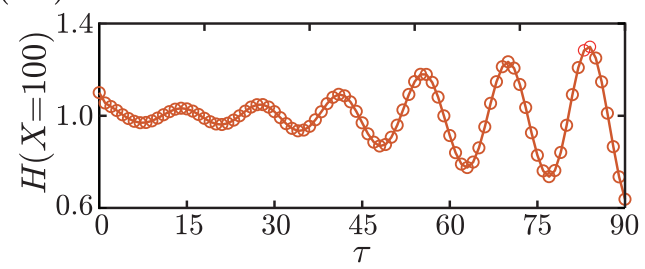

FIG. 7. Spatiotemporal evolution of a local disturbance by numerical simulations of the full evolution equation when $\alpha=\pi / 6$. (a1) A convectively unstable flow forms at Ma $=0$. (b1) An absolutely unstable flow forms at $\mathrm{Ma}=5$. The black straight lines in (a1) and (b1) depict the edges of the wave packet. (a2), (b2) The temporal evolution of the disturbance at its origin $X=100$, corresponding to (a1), (b1), respectively. Here, $\mathrm{We}=10, \mathrm{Bi}=1$, and $\mathrm{Re}=1$.

terminate the simulation before the disturbance periodically enters the computational domain from the upstream.

To clearly identify the signs of $V_{f}^{+}$and $V_{f}^{-}$, the spatiotemporal evolutions of the seeded disturbance are presented in the form of waterfall curves, where the film thickness profile $H(X)$ is sketched at different time instants $\tau$. The corresponding plots of the seeded disturbance for $\alpha=\pi / 6$ with $\mathrm{Ma}=0$ and $\mathrm{Ma}=5$ from the numerical solutions of Eq. (21) are shown in Figs. 7(a1) and 7(b1), respectively. For the set of $\alpha=\pi / 6$ and $\mathrm{Ma}=0$, as time evolves, the imposed localized excitation increases and sweeps towards downstream $\left(V_{f}^{+}>0\right.$ and $\left.V_{f}^{-}>0\right)$, with a flat, stable interface being left in the upstream region, demonstrating the flow is convectively unstable [see Fig. 7(a1)]. This observation is qualitatively in agreement with the recent experimental finding and theoretical modeling of an isothermal film flow under an inclined plane, where the flow is convectively unstable and no drops form in the laboratory frame for a large inclination angle [5,29]. When the substrate is heated, as time evolves, the imposed localized excitation is rapidly amplified and the disturbance not only propagates downstream $\left(V_{f}^{+}>0\right)$ but also upstream $\left(V_{f}^{-}<0\right)$, as shown in Fig. 7(b1). Such a phenomenon demonstrates the flow is absolutely unstable. In addition, to further distinguish the absolute and convective nature of the instability, we resort to the time-dependent amplitudes of the imposed impulse, as shown in Figs. 7(a2) and 7(b2). When $\mathrm{Ma}=0$, the initial disturbance imposed at its origin $(X=100)$ decays with time, becoming negligible at $\tau \approx 40$, further suggesting that the instability is convective [Fig. 7(a2)]. Conversely, when $\mathrm{Ma}=5$, the growing disturbance despite the presence of the mean flow in the laboratory frame indicates the absolute nature of the instability [Fig. 7(b2)].

For a weak inclination with $\alpha=\pi / 12$, Figs. 8(a1) and 8(a2) show the absolute instability of the isothermal film flow with $V_{f}^{+}>0, V_{f}^{-}<0$ and the accompanying time-dependent growing amplitude at the origin, respectively. On the contrary, for a strongly cooled film with $\mathrm{Ma}=-5$, the thermocapillary stress drives the absolute instability towards the convective instability $\left(V_{f}^{+}>0\right.$, 
(a1)

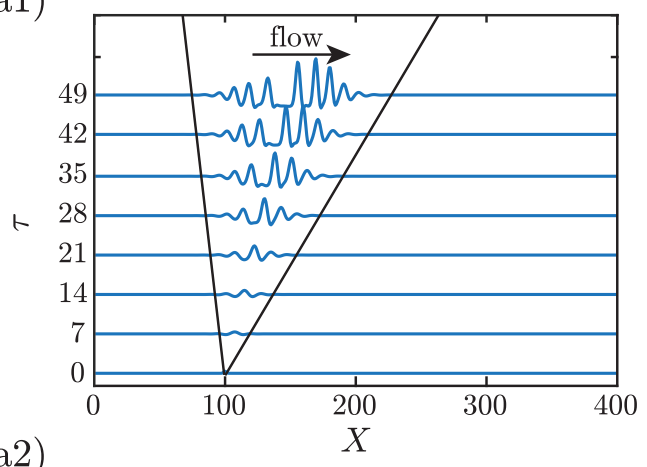

(a2)

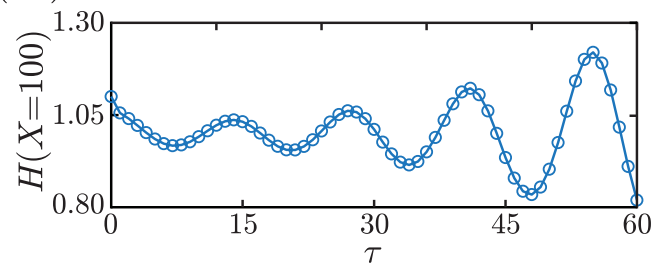

(b1)

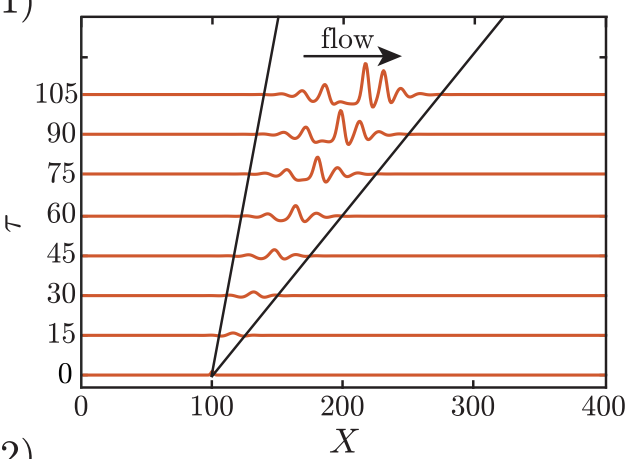

(b2)

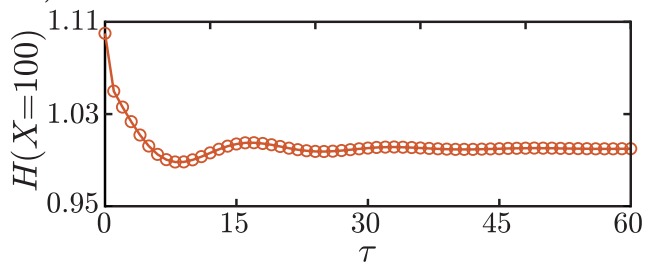

FIG. 8. Similar to Fig. 7 but for an inclination angle $\alpha=\pi / 12$ rather than $\alpha=\pi / 6$. (a) An absolutely unstable flow when $\mathrm{Ma}=0$ and (b) a convectively unstable flow when $\mathrm{Ma}=-5$.

$V_{f}^{-}>0$, and the time-dependant decaying amplitude at its origin), as shown in Figs. 8(b1) and $8(\mathrm{~b} 2)$.

To briefly conclude, the thermocapillary stress developed in a heated film enhances the absolute instability and suppresses the convective instability. In contrast, the developed stress in a cooled film acts oppositely by suppressing the absolute instability and enhancing the convective instability. The numerical solutions to the full nonlinear evolution Eq. (21) agree with the predictions from linear stability analysis for both isothermal and nonisothermal cases. More importantly, the demonstrated ability of the thermocapillary stress to modulate the spatiotemporal stability of the RT instability emphasizes the possibility of manipulating the formation of suspending droplets for industrial applications. Note that our study using the long-wave assumption do not intend to capture droplets pinching off from the substrate $[47,48]$.

\section{CONCLUSIONS AND DISCUSSION}

In summary, we have systematically investigated the thermocapillary effect on the RT instability of viscous liquid films under a temperature-controlled (uniformly heated or cooled) inclined substrate. A long-wave model applicable for small Reynolds number Re is used to derive the evolution equation for the flow dynamics. Based on the evolution equation, we examine the temporal and spatiotemporal stability of the system for heated and cooled substrates, aiming to identify the absolute and convective instability. Temporal stability analysis shows the persisting existence of the RT instability in the absence of the thermocapillary stress. This inherent instability is further augmented by the thermocapillary stress for a heated substrate. The scenario reverses on a cooled substrate, where the thermocapillary stress stabilizes the film and suppresses the RT instability. This observation is consistent with an earlier work on liquid films under a cooled, horizontal plane [26]. In particular, the RT instability can be completely suppressed when a composite Marangoni number, $\mathrm{Ma}^{*}=\frac{3 \mathrm{MaBi}}{2(1+\mathrm{Bi})^{2}}$, is less than a critical value $\mathrm{Ma}_{1}^{*}=-\left(\cot \alpha+\frac{2}{5} \mathrm{Re}\right)$. The fair agreement between the results from the long-wave evolution equation and those from the linearized NS equation further validates the long-wave model used in the present study.

The absolute and convective nature of the temporal instability is further characterized in the laboratory frame of reference by performing the spatiotemporal stability analysis. By rescaling the 
involved variables, we obtain a convenient form of the dispersion relation to evaluate the transition from the convective to absolute instability in the $\mathrm{Ma}-\alpha$ parameter space. For a given inclination angle $\alpha$, we show that thermocapillary stress promotes the absolute instability and suppresses the convective instability when the film is heated, which, however, suppresses the absolute instability and promotes the convective instability when the film is cooled. More importantly, an exact expression for a threshold value of the composite Marangoni number Ma* is derived in the form of $\mathrm{Ma}_{2}^{*}=\left[\frac{9}{4}(-17+7 \sqrt{7}) \mathrm{We}\right]^{\frac{1}{3}}-\left(\cot \alpha+\frac{2}{5} \mathrm{Re}\right)$, beyond which the thermocapillarity will drive the flow to transit from the convective instability to the absolute instability. In the end, we perform transient numerical simulations of the full evolution equation. The numerical results agree well with the predictions from the temporal and spatiotemporal stability analysis.

Our work derives analytically two critical composite Marangoni numbers, $\mathrm{Ma}_{1}^{*}$ and $\mathrm{Ma}_{2}^{*}$, which delineate the stable, the convectively unstable, and the absolutely unstable regions for a nonisothermal film flowing under an inclined substrate. These results provide theoretical guidance for controlling or utilizing the RT instability in industrial applications, such as in designing complex soft materials of a particular pattern [49].

It is worth noting that the first-order long-wave model, the Benny-type equation we derive, can describe the main characteristics of the low-Reynolds-number flows in this work, as confirmed by the numerical solutions to the linearized NS equation. However, to extend this study into flows with large inertia effect, we need to resort to higher-order models, such as the second-order model, i.e., the well-known weighted residual integral boundary layer model $[35,36]$ as demonstrated by Scheid et al [29]. As a final remark, we would also like to note that the thin liquid film considered in this work is two dimensional. Recently, some remarkable patterns arising from the three-dimensional effects have been reported for isothermal films [50-52], such as the emergence of rivulets in response to streamwise-invariant sinusoidal initial conditions [52]. Therefore, it would be interesting to explore in future how the thermocapillary effect influences the three-dimensional RT instability of flowing liquid films.

\section{ACKNOWLEDGMENTS}

We acknowledge the anonymous referees for their constructive and valuable comments. L.Z. acknowledges the start-up grant (No. R-265-000-696-133) given by the National University of Singapore.

\section{APPENDIX A: DERIVATION OF LEADING-ORDER AND FIRST-ORDER SOLUTIONS}

In this work, we only consider the first-order $\mathrm{O}(\epsilon)$ model, where the governing equations, Eq. (12), and the associated boundary conditions, Eqs. (13) and (14), become

$$
\begin{aligned}
U_{X}+W_{Z} & =0 \\
\epsilon \operatorname{Re}\left(U_{\tau}+U U_{X}+W U_{Z}\right) & =-\epsilon P_{X}+U_{Z Z}+1, \\
-P_{z}+\epsilon W_{Z Z}+\cot \alpha & =0 \\
\theta_{Z Z} & =0 .
\end{aligned}
$$

At $Z=0$,

$$
U=0, \quad W=0, \quad \theta=1,
$$

and at $Z=H$,

$$
\begin{aligned}
& P=-2 \epsilon H_{X} U_{Z}+2 \epsilon W_{Z}-\epsilon^{2} \mathrm{We}_{X X}, \\
& U_{Z}=-\epsilon \mathrm{Ma} \theta_{X} \\
& \theta_{Z}+\operatorname{Bi} \theta=0
\end{aligned}
$$


As mentioned, all variables $[U, V, P, \theta]$ are expanded in power series of $\epsilon$,

$$
[U, V, P, \theta]=\left[U^{(0)}, V^{(0)}, P^{(0)}, \theta^{(0)}\right]+\epsilon\left[U^{(1)}, V^{(1)}, P^{(1)}, \theta^{(1)}\right]+O\left(\epsilon^{2}\right),
$$

which are inserted into Eqs. (A1)-(A3) to yield the leading-order and the first-order governing equations.

\section{The leading-order solution}

The leading-order governing equations are

$$
U_{X}^{(0)}+W_{Z}^{(0)}=0, \quad U_{Z Z}^{(0)}=-1, \quad P_{Z}^{(0)}=\cot \alpha, \quad \theta_{Z Z}^{(0)}=0,
$$

with the following boundary conditions: at $Z=0$,

$$
U^{(0)}=0, \quad W^{(0)}=0, \quad \theta^{(0)}=1,
$$

and at $Z=H$,

$$
P^{(0)}=-\epsilon^{2} \mathrm{We} H_{X X}, \quad U_{Z}^{(0)}=0, \quad \theta_{Z}^{(0)}+\mathrm{Bi} \theta^{(0)}=0 .
$$

After solving the above equations, we can get the leading-order velocities $\left[U^{(0)}, W^{(0)}\right]$, pressure $P^{(0)}$, and temperature $\theta^{(0)}$ :

$$
\begin{aligned}
U^{(0)} & =-\frac{1}{2} Z^{2}+H Z, \\
W^{(0)} & =-\frac{1}{2} H_{X} Z^{2}, \\
P^{(0)} & =\cot \alpha(Z-H)-\epsilon^{2} \mathrm{We} H_{X X}, \\
\theta^{(0)} & =1-\frac{\mathrm{BiZ}}{1+\mathrm{Bi} H},
\end{aligned}
$$

where the leading-order interfacial temperature $\theta^{\mathrm{i},(0)}=\left.\theta^{(0)}\right|_{Z=H}=\frac{1}{1+\mathrm{BiH}}$.

\section{The first-order solution}

Similarly, the first-order governing equations are

$$
\begin{aligned}
& U_{X}^{(1)}+W_{Z}^{(1)}=0, \\
& U_{Z Z}^{(1)}=\operatorname{Re}\left(U_{\tau}^{(0)}+U^{(0)} U_{X}^{(0)}+W^{(0)} U_{Z}^{(0)}\right)+P_{X}^{(0)}, \\
& P_{Z}^{(1)}=W_{Z Z}^{(0)}, \\
& \theta_{Z Z}^{(1)}=0 .
\end{aligned}
$$

The boundary conditions are, at $Z=0$,

$$
U^{(1)}=0, \quad W^{(1)}=0, \quad \theta^{(1)}=0,
$$

and at $Z=H$,

$$
\begin{aligned}
& P^{(1)}=-2 H_{X} U_{Z}^{(0)}+2 W_{Z}^{(0)}, \\
& U_{Z}^{(1)}=-\operatorname{Ma} \theta_{X}^{(0)}, \\
& \theta_{Z}^{(1)}+\operatorname{Bi} \theta^{(1)}=0 .
\end{aligned}
$$


By solving the above equations, we derive the first-order streamwise velocity $U^{(1)}$ and pressure $P^{(1)}$ :

$$
\begin{aligned}
U^{(1)}= & \left(-\cot \alpha H_{X}-\epsilon^{2} \mathrm{We} H_{X X X}\right)\left(\frac{Z^{2}}{2}-H Z\right)+\frac{\operatorname{MaBi} H_{X}}{(1+\mathrm{Bi} H)^{2}} Z \\
& +\operatorname{Re}\left[\left(\frac{Z^{4}}{24}-\frac{H^{3} Z}{6}\right) H H_{X}+\left(\frac{Z^{3}}{6}-\frac{H^{2} Z}{2}\right) H_{\tau}\right], \\
P^{(1)}= & -(Z-H) H_{X}-2 H H_{X} .
\end{aligned}
$$

\section{APPENDIX B: THE LINEARIZED NS EQUATIONS}

We here present the details of deriving and solving the linearized NS equations. Following the long-wave analysis, Pe and $\frac{\mathrm{Ma}}{\mathrm{We}}$ in Eqs. (8d) and (10a) are assumed to be small and not considered. Accordingly, the interfacial temperature $\theta^{\mathrm{i}}$ can be separately solved as

$$
\theta^{\mathrm{i}}=\frac{1}{1+\mathrm{Bi} H}
$$

The standard normal-mode decomposition is applied, and all variables $[U, W, P, H]$ are perturbed by infinitesimal harmonic perturbations in the form of

$$
[U, W, P, H]=[\bar{U}, \bar{W}, \bar{P}, \bar{H}]+[\hat{U}, \hat{W}, \hat{P}, \hat{H}] \exp (i k X+\omega \tau),
$$

where $[\bar{U}, \bar{W}, \bar{P}, \bar{H}]$ represent the base states, and $[\hat{U}, \hat{W}, \hat{P}, \hat{H}]$ denote the Fourier amplitudes of the perturbation. Here $k$ is the wave number and $\omega$ is the complex growth rate. The base states of the velocity and pressure are

$$
\bar{U}=-\frac{1}{2} Z^{2}+Z, \quad \bar{W}=0, \quad \bar{P}=-\cot \alpha(1-Z) .
$$

By linearizing the governing system Eqs. (8a)-(8c) with respect to the base states [Eq. (B3)], we obtain the following eigenvalue system:

$$
\begin{aligned}
i k \hat{U}+\mathrm{D} \hat{W} & =0, \\
\operatorname{Re}(\omega \hat{U}+i k \bar{U} \hat{U}+\mathrm{D} \bar{U} \hat{W}) & =-i k \hat{P}+\left(\mathrm{D}^{2}-k^{2}\right) \hat{U}, \\
\operatorname{Re}(\omega \hat{W}+i k \bar{U} \hat{W}) & =-\mathrm{D} \hat{P}+\left(\mathrm{D}^{2}-k^{2}\right) \hat{W},
\end{aligned}
$$

where the operator $\mathrm{D}=d / d Z$. At the substrate surface $(Z=0)$, the linearized boundary conditions, Eq. (9), become

$$
\hat{U}=0, \quad \hat{W}=0 .
$$

At the liquid-air interface $(Z=H)$, the linearized boundary conditions, Eqs. (10a), (10b), and (10d), are projected to $Z=1$ by Taylor expansions, yielding

$$
\begin{aligned}
& \hat{P}+\cot \alpha \hat{H}+2 i k \mathrm{D} \bar{U} \hat{H}-2 \mathrm{D} \hat{W}-k^{2} \mathrm{We} \hat{H}=0, \\
& \mathrm{D} \hat{U}+i k \hat{W}=i k \frac{\mathrm{MaBi}}{(1+\mathrm{Bi})^{2}} \hat{H}+\hat{H}, \\
& \omega \hat{H}+i k \bar{U} \hat{H}=\hat{W},
\end{aligned}
$$

where all $O\left(H_{X}^{2}\right)$ terms are omitted for simplification. The above eigenvalue problem is solved by a Chebyshev collocation method [53,54], leading to the dispersion relation curves shown in Fig. 2. Moreover, we examine an additional case with a high Weber number, i.e., We $=100$, as illustrated in Fig. 9. It indicates that when the capillary effect is strong, the cutoff wave number $k_{c}$ and the most unstable mode $k_{m}$ calculated from the long-wave equation quantitatively agree with those from the linearized NS equation. 


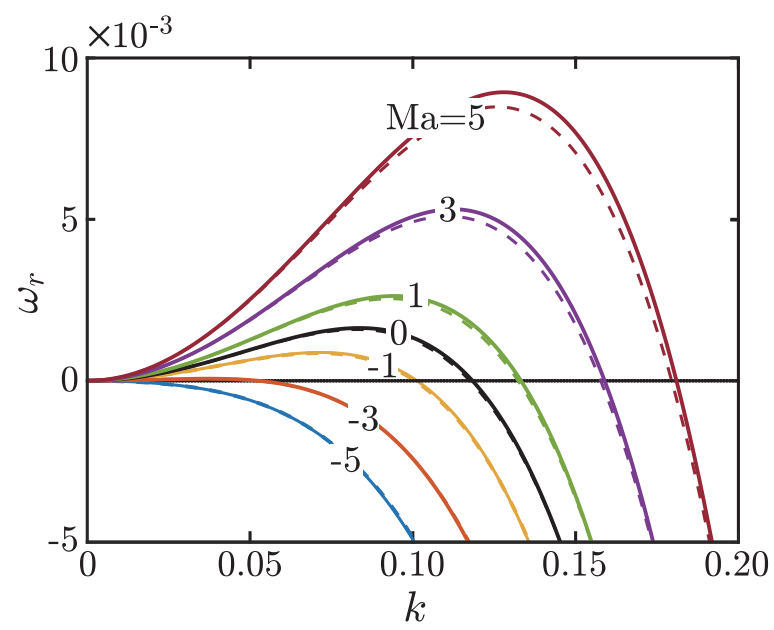

FIG. 9. Temporal growth rates $\omega_{r}$ vs wave number $k$ for a strong capillary effect $(\mathrm{We}=100)$ with different Ma values. Solid and dashed lines denote the results from the linearized long-wave model and the linearized NS equations, respectively. It should be noted that the solid and dashed lines almost overlap with each other for the cases of $\mathrm{Ma}=-1,-3$, and -5 . Here, $\alpha=\pi / 4, \mathrm{Bi}=1$, and $\mathrm{Re}=1$.

[1] L. Rayleigh, Investigation of the character of the equilibrium of an incompressible heavy fluid of variable density, Proc. Lond. Math. Soc. s1-14, 170 (1882).

[2] G. I. Taylor, The instability of liquid surfaces when accelerated in a direction perpendicular to their planes (I), Proc. R. Soc. Lond. A 201, 192 (1950).

[3] D. Lewis, The instability of liquid surfaces when accelerated in a direction perpendicular to their planes (II), Proc. R. Soc. Lond. A 202, 81 (1950).

[4] J. L. Vinningland, Ø. Johnsen, E. G. Flekkøy, R. Toussaint, and K. J. Måløy, Granular Rayleigh-Taylor Instability: Experiments and Simulations, Phys. Rev. Lett. 99, 048001 (2007).

[5] P.-T. Brun, A. Damiano, P. Rieu, G. Balestra, and F. Gallaire, Rayleigh-Taylor instability under an inclined plane, Phys. Fluids 27, 084107 (2015).

[6] R. G. Evans, A. J. Bennett, and G. J. Pert, Rayleigh-Taylor Instabilities in Laser-Accelerated Targets, Phys. Rev. Lett. 49, 1639 (1982).

[7] R. Kaita, L. Berzak, D. Boyle, T. Gray, E. Granstedt, G. Hammett, C. M. Jacobson, A. Jones, T. Kozub, H. Kugel et al., Experiments with liquid metal walls: Status of the lithium tokamak experiment, Fusion Eng. Des. 85, 874 (2010).

[8] L. L. Barannyk, D. T. Papageorgiou, and P. G. Petropoulos, Suppression of Rayleigh-Taylor instability using electric fields, Maths Comput. Simul. 82, 1008 (2012).

[9] R. Cimpeanu, D. T. Papageorgiou, and P. G. Petropoulos, On the control and suppression of the RayleighTaylor instability using electric fields, Phys. Fluids 26, 022105 (2014).

[10] R. J. Tomlin, R. Cimpeanu, and D. T. Papageorgiou, Instability and dripping of electrified liquid films flowing down inverted substrates, Phys. Rev. Fluids 5, 013703 (2020).

[11] G. Wolf, Dynamic Stabilization of the Interchange Instability of a Liquid-Gas Interface, Phys. Rev. Lett. 24, 444 (1970).

[12] V. Lapuerta, F. J. Mancebo, and J. M. Vega, Control of Rayleigh-Taylor instability by vertical vibration in large aspect ratio containers, Phys. Rev. E 64, 016318 (2001).

[13] E. Talib and A. Juel, Instability of a viscous interface under horizontal oscillation, Phys. Fluids 19, 092102 (2007). 
[14] E. Sterman-Cohen, M. Bestehorn, and A. Oron, Rayleigh-Taylor instability in thin liquid films subjected to harmonic vibration, Phys. Fluids 29, 052105 (2017).

[15] D. Rannacher and A. Engel, Suppressing the Rayleigh-Taylor instability with a rotating magnetic field, Phys. Rev. E 75, 016311 (2007).

[16] A. Pöhlmann, R. Richter, and I. Rehberg, Unravelling the Rayleigh-Taylor instability by stabilization, J. Fluid Mech. 732, R3 (2013).

[17] K. A. Baldwin, M. M. Scase, and R. J. Hill, The inhibition of the Rayleigh-Taylor instability by rotation, Sci. Rep. 5, 11706 (2015).

[18] P. H. Trinh, H. Kim, N. Hammoud, P. D. Howell, S. J. Chapman, and H. A. Stone, Curvature suppresses the Rayleigh-Taylor instability, Phys. Fluids 26, 051704 (2014).

[19] G. Balestra, P.-T. Brun, and F. Gallaire, Rayleigh-Taylor instability under curved substrates: An optimal transient growth analysis, Phys. Rev. Fluids 1, 083902 (2016).

[20] S. Alqatari, T. E. Videbæk, S. R. Nagel, A. Hosoi, and I. Bischofberger, Confinement-induced stabilization of the Rayleigh-Taylor instability and transition to the unconfined limit, Sci. Adv. 6, eabd6605 (2020).

[21] M. Fermigier, L. Limat, J. Wesfreid, P. Boudinet, and C. Quilliet, Two-dimensional patterns in RayleighTaylor instability of a thin layer, J. Fluid Mech. 236, 83 (1992).

[22] J. R. Lister, J. M. Rallison, and S. J. Rees, The nonlinear dynamics of pendent drops on a thin film coating the underside of a ceiling, J. Fluid Mech. 647, 239 (2010).

[23] D. Sharp, An overview of Rayleigh-Taylor instability, Physica D 12, 3 (1984).

[24] S. H. Davis, Thermocapillary instabilities, Annu. Rev. Fluid Mech. 19, 403 (1987).

[25] R. Craster and O. Matar, Dynamics and stability of thin liquid films, Rev. Mod. Phys. 81, 1131 (2009).

[26] R. J. Deissler and A. Oron, Stable Localized Patterns in thin Liquid Films, Phys. Rev. Lett. 68, 2948 (1992).

[27] J. M. Burgess, A. Juel, W. D. McCormick, J. B. Swift, and H. L. Swinney, Suppression of Dripping from a Ceiling, Phys. Rev. Lett. 86, 1203 (2001).

[28] A. Alexeev and A. Oron, Suppression of the Rayleigh-Taylor instability of thin liquid films by the Marangoni effect, Phys. Fluids 19, 082101 (2007).

[29] B. Scheid, N. Kofman, and W. Rohlfs, Critical inclination for absolute/convective instability transition in inverted falling films, Phys. Fluids 28, 044107 (2016).

[30] S. Kalliadasis, C. Ruyer-Quil, B. Scheid, and M. G. Velarde, Falling Liquid Films (Springer Science \& Business Media, New York, 2011), Vol. 176.

[31] T. L. Bergman, F. P. Incropera, D. P. DeWitt, and A. S. Lavine, Fundamentals of Heat and Mass Transfer (John Wiley \& Sons, New York, 2011).

[32] Y. Chao and Z. Ding, Thermocapillary thin-film flows on a compliant substrate, Phys. Rev. E 99, 043101 (2019).

[33] A. Pumir, P. Manneville, and Y. Pomeau, On solitary waves running down an inclined plane, J. Fluid Mech. 135, 27 (1983).

[34] P. L. Kapitza and S. Kapitza, Wave flow of thin layers of a viscous fluid, Zh. Eksp. Teor. Fiz. 19, 105 (1949).

[35] C. Ruyer-Quil and P. Manneville, Improved modeling of flows down inclined planes, Eur. Phys. J. B 15, 357 (2000).

[36] C. Ruyer-Quil and P. Manneville, Further accuracy and convergence results on the modeling of flows down inclined planes by weighted-residual approximations, Phys. Fluids 14, 170 (2002).

[37] R. J. Briggs, Electron-Stream Interaction with Plasmas (MIT Press, Cambridge, 1964).

[38] A. Bers, Linear waves and instabilities, in Physique des Plasmas, edited by C. DeWitt and J. Peyraud (Gordon and Breach, New York, 1975).

[39] P. Huerre and P. A. Monkewitz, Local and global instabilities in spatially developing flows, Annu. Rev. Fluid Mech. 22, 473 (1990).

[40] Z. Ding, R. Liu, T. N. Wong, and C. Yang, Absolute instability induced by Marangoni effect in thin liquid film flows on vertical cylindrical surfaces, Chem. Eng. Sci. 177, 261 (2018). 
[41] C. Duprat, C. Ruyer-Quil, S. Kalliadasis, and F. Giorgiutti-Dauphiné, Absolute and Convective Instabilities of a Viscous Film Flowing Down a Vertical Fiber, Phys. Rev. Lett. 98, 244502 (2007).

[42] R. Liu and Z. Ding, Instabilities and bifurcations of liquid films flowing down a rotating fibre, J. Fluid Mech. 899, A14 (2020).

[43] S. Khanum and N. Tiwari, Gravity-driven thermoviscous liquid film down a heated or cooled vertical cylinder, Phys. Rev. Fluids 5, 094005 (2020).

[44] C. Canuto, M. Y. Hussaini, A. Quarteroni, and A. Thomas, Jr., Spectral Methods in Fluid Dynamics, Springer Series in Computations Physics (Springer Science \& Business Media, New York, 2012).

[45] C. W. Gear, Numerical Initial Value Problems in Ordinary Differential Equations (Prentice Hall PTR, Upper Saddle River, NJ, 1971).

[46] Y. Chao, Y. Lu, and H. Yuan, On reactive thin liquid films falling down a vertical cylinder, Int. J. Heat Mass Transfer 147, 118942 (2020).

[47] T.-S. Lin and L. Kondic, Thin films flowing down inverted substrates: Two dimensional flow, Phys. Fluids 22, 052105 (2010).

[48] N. Kofman, W. Rohlfs, F. Gallaire, B. Scheid, and C. Ruyer-Quil, Prediction of two-dimensional dripping onset of a liquid film under an inclined plane, Int. J. Multiphase Flow 104, 286 (2018).

[49] J. Marthelot, E. F. Strong, P. M. Reis, and P.-T. Brun, Designing soft materials with interfacial instabilities in liquid films, Nat. Commun. 9, 4477 (2018).

[50] A. Charogiannis, F. Denner, B. G. van Wachem, S. Kalliadasis, B. Scheid, and C. N. Markides, Experimental investigations of liquid falling films flowing under an inclined planar substrate, Phys. Rev. Fluids 3, 114002 (2018).

[51] G. Lerisson, P. G. Ledda, G. Balestra, and F. Gallaire, Instability of a thin viscous film flowing under an inclined substrate: Steady patterns, J. Fluid Mech. 898, A6 (2020).

[52] P. G. Ledda, G. Lerisson, G. Balestra, and F. Gallaire, Instability of a thin viscous film flowing under an inclined substrate: The emergence and stability of rivulets, J. Fluid Mech. 904, A23 (2020).

[53] P. J. Schmid and D. S. Henningson, Stability and Transition in Shear Flows (Springer, New York, 2001).

[54] Z. Ding and T. N. Wong, Falling liquid films on a slippery substrate with Marangoni effects, Int. J. Heat Mass Transfer 90, 689 (2015). 